\title{
Genomic heterogeneity: next-generation sequencing enables biomarker identification for hepatocellular carcinoma
}

\author{
"Clinicogenomic predictive models are necessary for tailored \\ treatment and individualized selection of the optimal agent matched \\ to a specific molecular landscape."
}

First draft submitted: 9 April 2017; Accepted for publication: 21 April 2017;

Published online: 12 July 2017

Keywords: biomarkers $\bullet$ cancer genome $\bullet$ hepatocellular carcinoma $\bullet$ next-generation sequencing $\bullet$ targeted NGS $\bullet$ whole-exome sequencing $\bullet$ whole-genome sequencing

Prognosis of hepatocellular carcinoma (HCC) remains poor. The slow progress in understanding the molecular mechanisms driving tumorigenesis, metastasis and therapeutic resistance explains the low rates of early detection and overall survival. This editorial summarizes the latest advances and challenges of next-generation sequencing (NGS) in developing robust biomarkers to predict the individualized risk and therapeutic response of HCCs.

The incidence and cancer-related death rates of HCC remain alarmingly high [1]. The modern screening programs for highrisk patients combine ultrasonographic imaging of the liver with measurement of serum $\alpha$-fetoprotein (AFP) levels every 6 months [2].

Currently, standardized complete surgical resection (R0) remains the only potentially curative therapeutic modality for HCC, which has reduced recurrence rates to approximately $55 \%$ and improved 5 years survival rates to $45 \%$ [3]. Despite the efforts toward developing effective adjuvant systemic therapy, no chemotherapeutic regimen or targeted drug has been approved [2]. Prognosis for the unresectable or metastatic disease is still grim, despite the availability of sorafenib and the expected approval of regorafenib, which prolong overall survival by a few months only [4].
The introduction of NGS providing evidence on genetic and genomic heterogeneity suggests an urgent need for the development of effective biomarkers enabling personalized prevention or treatment of HCC.

\section{Modern management of HCC}

Diagnosis of HCC is based upon imaging, as modern imaging advances have allowed for the accurate detection and staging of liver tumors, thus eliminating the need for routine presurgical biopsy. Diagnosis is made on the acquisition of the characteristic imaging pattern of HCC on a triple-phase CT or MRI scan, which features early arterial enhancement and washout in the venous phase. As mentioned, screening includes the use of liver ultrasound (US) and serum AFP measurement and is recommended for patients with cirrhosis, nonalcoholic fatty liver disease and hepatitis B carriers. Moreover, the presurgical staging workup further includes a hepatitis panel, liver function tests, baseline AFP levels, as well as total liver volume and functional liver remnant assessment [2].

The cornerstone of current treatment of $\mathrm{HCC}$ is R0 tumor resection through partial hepatectomy, which remains the only modality with curative potential. Patients meeting the United Network for Organ Sharing (UNOS) criteria can undergo orthotopic liver transplantation with remarkably low
Demosthenes E Ziogas 1,2, Efstathios G Lykoudis4, Dimitrios H Roukos ${ }^{* 1,3,5}$ \& Georgios K Glantzounis 3 ${ }^{1}$ Centre for Biosystems \& Genome Network Medicine, Ioannina University, Ioannina, Greece

2Department of Surgery, 'G. Hatzikosta' General Hospital, Ioannina, Greece ${ }^{3}$ Department of Surgery, Ioannina University Hospital, Ioannina, Greece ${ }^{4}$ Department of Plastic Surgery, Ioannina University Hospital, Ioannina, Greece ${ }^{5}$ Biomedical Research Foundation of the Academy of Athens (BRFAA), Athens, Greece

*Author for correspondence: Tel.: +30 2651005572 droukos@uoi.gr 
relapse and high progression-free and overall survival rates. In contrast to the majority of other malignancies, no effective adjuvant therapy currently exists in the case of HCC [2], despite extensive research efforts, especially in the field of targeted drug development. With the exception of the RESORCE trial on regorafenib, all other Phase III randomized controlled trials on experimental targeted agents have been negative, possibly due to the fact that none had implemented a biomarker-based patient selection protocol [5]. Lastly, the treatment of advanced HCC includes locoregional therapies, systemic targeted treatment with sorafenib and other chemotherapeutic regimens [2].

\section{Genome sequencing technologies}

Over the past decade, NGS has revolutionized biomedical research including biomarker identification. Indeed, definite evidence on genetic and genomic heterogeneity raises the need for biomarker-based personalized medicine [6-8]. The ability of NGS to identify molecular subgroups of patients enables the genomic classification of tumors irrespective of cancer type [9]. Progress in NGS integration into clinical studies is so rapid that targeted NGS has already achieved direct clinical implications, while whole-exome (WES) and whole-genome sequencing (WGS) have proven valid for the discovery of novel cancer driver genes and mutations [10].

\section{"Despite the efforts to prevent hepatic cancer, incidence rates continue to increase rapidly by more than $3 \%$ per year."}

\section{Umbrella \& basket studies}

The easy and low-cost conduction of targeted NGS on a panel of known genes can identify cancer driver genes involved in a specific (umbrella design) or multiple (basket design) cancer types [11]. Although there have been no umbrella and basket studies on HCC, highly promising findings have been reported for other cancer types [12,13].

\section{Whole-exome \& whole-genome NGS}

Most large-scale studies available have used WES for the identification of genes involved in tumorigenesis, while WGS can additionally detect large structural genomic alterations, such as copy number alterations and chromosomal rearrangements, at the expense of much higher complexity and cost. The rapid progress of bioinformatics allows for large-scale WGS studies elucidating on how noncoding sequence variations contribute to tumorigenesis apart from protein-coding genes, which may improve our understanding on the origins and development of HCC.
In two large WES projects on HCC, environmental risk factors, such as alcohol and viral hepatitis were associated with specific cancer driver genes (CTNNB1, TP53, AXINI) [14,15]. In contrast to these studies focusing on the effect of gene-environment interplay on tumorigenesis, other studies are on the identification of prognostic factors in the treatment setting. For instance, a study on $231 \mathrm{HCC}$ patients has been published by Ahn et al. has reported the potential implication of RB1 as a prognostic molecular biomarker after HCC resection [16]. Moreover, a handful of smaller WES studies have been published, providing promising findings towards the development of prognostic, predictive and therapeutic biomarkers, but they require confirmation by sizeable validation studies [5].

Recently, Fujimoto et al. reported a WGS analysis on 300 primary liver cancers, 268 of which were HCCs, 8 mixed HCCs and intrahepatic cholangiocarcinomas and 24 intrahepatic cholangiocarcinomas [7]. Three novel cancer driver genes were identified (ASH1L, NCOR1 and MACROD2) with significant validity, due to this study being the largest WGS report currently available on HCC. Furthermore, the authors identified an interplay between genetic characteristics and environmental risk factors with screening and preventive potential. Moreover, Fujimoto and colleagues, exploiting the capability of WGS to identify noncoding variants, have identified point mutations, structural variations and virus integrations in noncoding regions. Prognostic biomarkers with potential clinical implications were identified in this comprehensive WGS analysis. More specifically, mutations of driver genes such as TP53, CTNNB1, CDKN2A, MACROD2 and ARID2 or PBRM1 were significantly correlated with disease-free survival.

\section{Risk assessment: environmental-genetic interactions}

Despite the efforts to prevent hepatic cancer, incidence rates continue to increase rapidly by more than $3 \%$ per year [1], suggesting the need to develop an effective preventive strategy. Moreover, the rates of late diagnosis at advanced stages is $45 \%$, while these for localized disease are limited to $43 \%$ [1]. These facts indicate modest progress in the development of effective screening programs for the early detection of the disease. These data are not surprising considering that current guidelines for screening of high-risk individuals are based on environmental risk factors and clinical findings only. The recent NGS findings, revealing the genetic contribution to tumorigenesis, open new roads to early detection and prevention.

Three genes (CTNNB1, TP53 and AXINI) have been associated with environmental risk factors for HCC. Larger studies are required to detect novel genes inter- 
playing with external risk factors and for a more individualized screening approach. However, according to Lawrence's recommendations on appropriately sized samples, large-scale validation studies are needed. Should all these studies provide positive results on genetic-environmental interactions underlying tumorigenesis, the cost-effectiveness of CT scanning could be evaluated within clinical trials and, possibly, be added to the screening program to increase the rates of early-stage disease diagnosis.

\section{Future perspective \& conclusion}

Molecular classification and clinicogenomic models predicting intrinsic and acquired therapeutic resistance represent the most attractive emerging directions for biomedical research. Indeed, the potential identification of the origins and molecular classes of HCC [17] opens new therapeutic roads. The identification of the molecular mechanisms underlying tumorigenesis shapes the development of novel agents for primary prevention by disrupting progression to tumorigenesis. Following disease diagnosis, establishment of molecular and genomic subgroups forms the basis of future discovery of drugs targeting these specific subpopulations.

Although there has yet been no molecular taxonomy for HCC, a prime paradigm on how to utilize WES and WGS analyses for molecular subtyping has recently been reported for pancreatic ductal adenocarcinoma. Bailey et al. [18] have performed a comprehensive genomic analysis of 456 pancreatic ductal adenocarcinoma patients. Whole-genome sequencing was performed on 179 and WES on 204 tumor samples. Using RNA sequencing (RNA-seq) and array-based mRNA expression profiles, the researchers defined four subtypes: squamous, pancreatic progenitor, immunogenic and aberrantly differentiated endocrine exocrine. These tumor subsets correlated with histopathological characteristics and could bare prognostic significance. Toward this direction, a similar classification could be developed for HCC by using WES, WGS, RNA-seq and mRNA expression profiling.

The development of robust biomarkers and predictive models could overcome the unmet need of primary and secondary therapeutic resistance. Rapid advances in NGS technologies and methods allow for an opti-

\section{References}

Papers of special note have been highlighted as: • of interest;

• of considerable interest

1 Siegel RL, Miller KD, Jemal A. Cancer Statistics, 2017. CA Cancer J. Clin. 67(1), 7-30 (2017).

2 National Comprehensive Cancer Network (2017). www.nccn.org/

3 Tabrizian P, Jibara G, Shrager B, Schwartz M, Roayaie S. Recurrence of hepatocellular cancer after resection: patterns, mistic view on the realization of the goals of predictive cancer medicine. However, multiple challenges still remain. Precise predictive models require valid documentation and analysis of clinical, genomic, molecular and transcriptomic data before and after systemic treatment, as well as over the disease course, derived from well-designed and large-scale studies [19].

Indeed, dynamic evolution and emergence of distinct genomic subclones [20] in response to systemic chemotherapy and/or targeted therapy occurs often [21]. Therefore, dynamic predictive models are required in order to assess the comprehensive intrapatient variability, including intratumor heterogeneity, circulating genomic subclones and genomic alterations in relapsed (RT) or metastatic tumors (MT). Exciting breakthrough NGS applications have been developed to evaluate intratumor heterogeneity by multiregional tumor NGS analysis [22], circulating genomic subclones by noninvasive serial cell-free DNA NGS [23] and the genomic landscape of the RTs/MTs by fineneedle aspiration NGS or biopsies from the resected RTs/MTs.

In summary, emerging evidence on extensive tumor heterogeneity does not only explain current negative Phase III trials on new agents for HCC but further underlies the need for the development of predictive biomarkers toward effective personalized treatment. Integration of WES, WGS and transcriptomics into large validation studies could enable the discovery of novel druggable mutations, forming the basis of biomarker-directed drug development. Clinicogenomic predictive models are necessary for tailored treatment and individualized selection of the optimal agent matched to a specific molecular landscape.

\section{Financial \& competing interests disclosure}

The authors have no relevant affiliations or financial involvement with any organization or entity with a financial interest in or financial conflict with the subject matter or materials discussed in the manuscript. This includes employment, consultancies, honoraria, stock ownership or options, expert testimony, grants or patents received or pending, or royalties.

No writing assistance was utilized in the production of this manuscript.

treatments, and prognosis. Ann. Surg. 261(5), 947-955 (2015).

4 Bruix J, Qin S, Merle P et al. Regorafenib for patients with hepatocellular carcinoma who progressed on sorafenib treatment (RESORCE): a randomised, double-blind, placebo-controlled, Phase III trial. Lancet 389(10064), 56-66 (2017).

5 Kyrochristos ID, Glantzounis GK, Ziogas DE et al. From clinical standards to translating next-generation sequencing 
research into patient care improvement for hepatobiliary and pancreatic cancers. Int. J. Mol. Sci. 18(1), (2017).

6 Lawrence MS, Stojanov P, Mermel CH et al. Discovery and saturation analysis of cancer genes across 21 tumour types. Nature 505(7484), 495-501 (2014).

7 Fujimoto A, Furuta M, Totoki Y et al. Whole-genome mutational landscape and characterization of noncoding and structural mutations in liver cancer. Nat. Genet. 48(5), 500-509 (2016).

8 Nik-Zainal S, Davies H, Staaf J et al. Landscape of somatic mutations in 560 breast cancer whole-genome sequences. Nature 534(7605), 47-54 (2016).

9 Stratton MR, Campbell PJ, Futreal PA. The cancer genome. Nature 458(7239), 719-724 (2009).

10 Swanton C, Soria JC, Bardelli A et al. Consensus on precision medicine for metastatic cancers: a report from the MAP conference. Ann. Oncol. 27(8), 1443-1448 (2016).

11 Biankin AV, Piantadosi S, Hollingsworth SJ. Patient-centric trials for therapeutic development in precision oncology. Nature 526(7573), 361-370 (2015).

12 Hofmann D, Nitz U, Gluz O et al. WSG ADAPT - adjuvant dynamic marker-adjusted personalized therapy trial optimizing risk assessment and therapy response prediction in early breast cancer: study protocol for a prospective, multicenter, controlled, non-blinded, randomized, investigator initiated Phase II/III trial. Trials 14, 261 (2013).

13 Hyman DM, Puzanov I, Subbiah V et al. Vemurafenib in multiple nonmelanoma cancers with BRAF V600 mutations. N. Engl. J. Med. 373(8), 726-736 (2015).

14 Schulze K, Imbeaud S, Letouze E et al. Exome sequencing of hepatocellular carcinomas identifies new mutational signatures and potential therapeutic targets. Nat.
Genet. 47(5), 505-511 (2015).

15 Totoki Y, Tatsuno K, Covington KR et al. Trans-ancestry mutational landscape of hepatocellular carcinoma genomes. Nat. Genet. 46(12), 1267-1273 (2014).

16 Ahn SM, Jang SJ, Shim JH et al. Genomic portrait of resectable hepatocellular carcinomas: implications of RB1 and FGF19 aberrations for patient stratification. Hepatology 60 (6), 1972-1982 (2014).

17 Sia D, Villanueva A, Friedman SL, Llovet JM. Liver cancer cell of origin, molecular class, and effects on patient prognosis. Gastroenterology 152(4), 745-761 (2016).

18 Bailey P, Chang DK, Nones K et al. Genomic analyses identify molecular subtypes of pancreatic cancer. Nature 531(7592), 47-52 (2016).

19 Biankin AV. The road to precision oncology. Nat. Genet. 49(3), 320-321 (2017).

20 Eirew P, Steif A, Khattra J et al. Dynamics of genomic clones in breast cancer patient xenografts at single-cell resolution. Nature 518(7539), 422-426 (2015).

21 Klein CA. Selection and adaptation during metastatic cancer progression. Nature 501(7467), 365-372 (2013).

22 Yates LR, Gerstung M, Knappskog S et al. Subclonal diversification of primary breast cancer revealed by multiregion sequencing. Nat. Med. 21(7), 751-759 (2015).

23 Frenel JS, Carreira S, Goodall J et al. Serial next-generation sequencing of circulating cell-free DNA evaluating tumor clone response to molecularly targeted drug administration. Clin. Cancer Res. 21(20), 4586-4596 (2015). 